Article

\title{
SOME PROPERTIES OF THE HERMITE POLYNOMIALS AND THEIR SQUARES AND GENERATING FUNCTIONS
}

\author{
FENG QI
}

Institute of Mathematics, Henan Polytechnic University, Jiaozuo City, Henan Province 454010, China; College of Mathematics, Inner Mongolia University for Nationalities, Tongliao City, Inner Mongolia Autonomous Region 028043, China; Department of Mathematics, College of Science, Tianjin Polytechnic University, Tianjin City 300387, China

\author{
BAI-NI GUO \\ School of Mathematics and Informatics, Henan Polytechnic University, Jiaozuo City, \\ Henan Province 454010, China
}

\begin{abstract}
In the paper, the authors consider the generating functions of the Hermite polynomials and their squares, present explicit formulas for higher order derivatives of the generating functions of the Hermite polynomials and their squares, which can be viewed as ordinary differential equations or derivative polynomials, find differential equations that the generating functions of the Hermite polynomials and their squares satisfy, and derive explicit formulas and recurrence relations for the Hermite polynomials and their squares.
\end{abstract}

\section{INTRODUCTION}

It is well known that the Hermite polynomials $H_{n}(x)$ can be generated by

$$
e^{2 x t-t^{2}}=\sum_{n=0}^{\infty} H_{n}(x) \frac{t^{n}}{n !}
$$

The first six Hermite polynomials $H_{n}(x)$ for $0 \leq n \leq 5$ are

$$
1, \quad 2 x, \quad 2\left(2 x^{2}-1\right), \quad 4 x\left(2 x^{2}-3\right), \quad 4\left(4 x^{4}-12 x^{2}+3\right), \quad 8 x\left(4 x^{4}-20 x^{2}+15\right) .
$$

In [3, p. 250], it was given that the squares $H_{n}^{2}(x)$ for $n \geq 0$ of the Hermite polynomials $H_{n}(x)$ can be generated by

$$
\frac{1}{\sqrt{1-t^{2}}} \exp \frac{2 x^{2} t}{1+t}=\sum_{n=0}^{\infty} \frac{H_{n}^{2}(x)}{2^{n}} \frac{t^{n}}{n !}
$$

E-mail addresses: qifeng618@gmail.com, qifeng618@hotmail.com, bai.ni.guo@gmail.com, bai.ni.guo@hotmail.com 2010 Mathematics Subject Classification. Primary 33C45; Secondary 11B83, 26A06, 26A09, 26A24, 33B10, 33C47, $34 \mathrm{~A} 05$.

Key words and phrases. Hermite polynomial; square; generating function; higher order derivative; differential equa-tion; derivative polynomial; explicit formula; recurrence relation.

This paper was typeset using $\mathcal{A}_{\mathcal{M}} \mathcal{S}$-IATEX. 
In [5], the equation (2) was reformulated as

$$
\frac{1}{\sqrt{1-t^{2}}} \exp \frac{x t}{1+t}=\sum_{n=0}^{\infty} H_{n}^{2}(\sqrt{x}) \frac{t^{n}}{n !} .
$$

Indeed, this is a typo and the corrected one should be

$$
\frac{1}{\sqrt{1-t^{2}}} \exp \frac{x t}{1+t}=\sum_{n=0}^{\infty} \frac{H_{n}^{2}(\sqrt{x / 2})}{2^{n}} \frac{t^{n}}{n !} .
$$

After inductively arguing for nine pages, it was obtained in [5, Theorem 1] that the ordinary differential equations

$$
F^{(n)}(t)=\left[\sum_{i=0}^{n} \sum_{j=n-i}^{2(n-i)} \frac{a_{i, j}(n, x)}{(1-t)^{i}(1+t)^{j}}\right] F(t)
$$

for $n \geq 0$ have the same solution

$$
F(t)=F(t, x)=\frac{1}{\sqrt{1-t^{2}}} \exp \frac{x t}{1+t},
$$

where

$$
\begin{gathered}
a_{0,0}(0, x)=1, \quad a_{1,0}(1, x)=\frac{1}{2}, \quad a_{0,1}(1, x)=-\frac{1}{2}, \\
a_{0,2}(1, x)=x, \quad a_{0, n}(n, x)=\left(-\frac{1}{2}\right)^{n}(2 n-1) ! !
\end{gathered}
$$

and

$$
\begin{aligned}
a_{i, j}(n, x)= & \sum_{k=0}^{2 n-j-2 i}\left(-\frac{1}{2}\right)^{k} \frac{(2 j-1) ! !}{(2 j-2 k-1) ! !} \\
& \times\left[\frac{2 i-1}{2} a_{i-1, j-k}(n-k-1, x)+x a_{i, j-k-2}(n-k-1, x)\right] .
\end{aligned}
$$

From [5, Theorem 1] mentioned above, Theorems 2 and 3 in [5], which can be corrected as

$$
\frac{H_{k+n}^{2}(\sqrt{x / 2})}{2^{k+n}}=\sum_{i=0}^{n} \sum_{j=n-i}^{2(n-i)} \sum_{p+q+r=k}(-1)^{q}\left(\begin{array}{c}
k \\
p, q, r
\end{array}\right)(i+p-1)_{p}(j+q-1)_{q} a_{i, j}(n, x) \frac{H_{r}^{2}(\sqrt{x / 2})}{2^{r}}
$$

and

$$
\frac{H_{n}^{2}(\sqrt{x / 2})}{2^{n}}=\sum_{i=0}^{n} \sum_{j=n-i}^{2(n-i)} a_{i, j}(n, x)
$$

for $k, n \geq 0$, were derived, where

$$
(x)_{n}= \begin{cases}x(x+1)(x+2) \ldots(x+n-1), & n \geq 1 \\ 1, & n=0\end{cases}
$$

denotes the rising factorial and

$$
\left(\begin{array}{c}
n \\
k_{1}, k_{2}, \ldots, k_{m}
\end{array}\right)=\frac{n !}{k_{1} ! k_{2} ! \cdots k_{m} !}
$$

is the multinomial coefficients. 
It is clear that the quantities $a_{i, j}(n, x)$ in [5] were expressed by a recurrent relation and can not be computed easily by hand and by computer softwares. We observe that, when $k=2 n-j-2 i$ and $i+j=n$, the quantity $a_{i, j-k-2}(n-k-1, x)$ in the recurrence relation (5) becomes

$$
\begin{gathered}
a_{i, j-k-2}(n-k-1, x)=a_{i, j-(2 n-j-2 i)-2}(n-(2 n-j-2 i)-1, x) \\
=a_{i, 2(i+j-n-1)}(2 i+j-n-1, x)=a_{i,-2}(i-1, x)
\end{gathered}
$$

which implies that Theorem 1, consequently Theorems 2 and 3 , in 5 , are wrong.

In this paper, we will reconsider the generating functions $e^{2 t x-t^{2}}$ and $F(t)=F(t, x)$ defined in (4), present explicit formulas for the $n$th derivatives of the functions $F(t)$ and $e^{2 t x-t^{2}}$, which can be viewed as ordinary differential equations or derivative polynomials [7, find more differential equations that the functions $F(t)$ and $e^{2 t x-t^{2}}$ satisfy, and derive explicit formulas and recurrence relations for the Hermite polynomials $H_{n}(x)$ and their squares $H_{n}^{2}(x)$.

The main results of this paper can be stated as the following theorems.

Theorem 1.1. For $n \geq 0$, the $n$th derivative of the function $F(t)=F(t, x)$ defined in (4) can be computed by

$$
\begin{aligned}
& \frac{\mathrm{d}^{n} F(t)}{\mathrm{d} t^{n}}=\left\{\frac { ( - 1 ) ^ { n } n ! } { ( 1 + t ) ^ { n } } \sum _ { m = 0 } ^ { n } \frac { ( - 1 ) ^ { m } } { m ! } \frac { 1 } { ( 1 + t ) ^ { m } } \left(\sum_{k=0}^{n-m} \frac{(-1)^{k}(1+t)^{k}}{2^{k}}\left(\begin{array}{c}
n-k-1 \\
m-1
\end{array}\right)\right.\right. \\
& \left.\left.\times\left[\frac{1}{t^{k}} \sum_{\ell=0}^{k} \frac{(2 \ell-1) ! ! 2^{\ell}}{\ell !}\left(\begin{array}{c}
\ell \\
k-\ell
\end{array}\right) \frac{t^{2 \ell}}{\left(1-t^{2}\right)^{\ell}}\right]\right) x^{m}\right\} F(t),
\end{aligned}
$$

where $\left(\begin{array}{l}0 \\ 0\end{array}\right)=1$ and $\left(\begin{array}{l}p \\ q\end{array}\right)=0$ for $q>p \geq 0$.

Theorem 1.2. For $n \geq 0$, the squares $H_{n}^{2}(x)$ of the Hermite polynomials $H_{n}(x)$ can be computed by

$$
H_{n}^{2}(x)=(-1)^{n} 2^{n} n ! \sum_{k=0}^{n}(-1)^{k} \frac{2^{k}}{k !}\left[\sum_{\ell=0}^{n-k} \frac{1+(-1)^{\ell}}{2} \frac{(\ell-1) ! !}{\ell ! !}\left(\begin{array}{c}
n-\ell-1 \\
k-1
\end{array}\right)\right] x^{2 k} .
$$

Theorem 1.3. For $n \geq 0$, the Hermite polynomials $H_{n}(x)$ can be computed by

$$
H_{n}(x)=(-1)^{n} \frac{n !}{2^{n}} \sum_{k=0}^{n}(-1)^{k} \frac{2^{2 k}}{k !}\left(\begin{array}{c}
k \\
n-k
\end{array}\right) x^{2 k-n}
$$

and the nth derivative of their generating function $e^{2 x t-t^{2}}$ can be computed by

$$
\frac{\mathrm{d}^{n} e^{2 x t-t^{2}}}{\mathrm{~d} t^{n}}=e^{2 x t-t^{2}} \frac{n !}{2^{n}} \sum_{k=0}^{n}(-1)^{k} \frac{2^{2 k}}{k !}\left(\begin{array}{c}
k \\
n-k
\end{array}\right)(t-x)^{2 k-n} .
$$

Theorem 1.4. For $n \geq 0$, the Hermite polynomials $H_{n}(x)$ and their derivatives $H_{n}^{\prime}(x)$ satisfy $H_{0}^{\prime}(x)=0$,

and

$$
H_{n}^{\prime}(x)=2 n H_{n-1}(x)
$$

for $n \in \mathbb{N}$. Consequently,

$$
H_{n}(x)=2 x H_{n-1}(x)-H_{n-1}^{\prime}(x)
$$

$$
H_{n}(x)=2 x H_{n-1}(x)-2(n-1) H_{n-2}(x)
$$

for $n \geq 2$. 
Theorem 1.5. For $n \geq 0$, the Hermite polynomials $H_{n}(x)$ satisfy the recurrence relations

$$
\sum_{k=0}^{n} \frac{1+(-1)^{n-k}}{2} \frac{2^{(n-k) / 2}}{(n-k) ! ! k !} H_{k}(x)=\frac{(2 x)^{n}}{n !}
$$

and

$$
\sum_{k=0}^{n}(-1)^{n-k}\left(\begin{array}{l}
n \\
k
\end{array}\right)(2 x)^{n-k} H_{k}(x)=\frac{1+(-1)^{n}}{2}(-2)^{n / 2} \frac{n !}{n ! !} .
$$

For $n \geq 0$, the squares $H_{n}^{2}(x)$ of the Hermite polynomials $H_{n}(x)$ satisfy the recurrence relations

$$
\sum_{k=0}^{n} \frac{1+(-1)^{n-k}}{2} \frac{(n-k-3) ! !}{(n-k) ! !(2 k) ! !} H_{k}^{2}(x)=(-1)^{n+1} \sum_{\ell=0}^{n} \frac{(-1)^{\ell}}{\ell !}\left(\begin{array}{c}
n-1 \\
\ell-1
\end{array}\right)\left(2 x^{2}\right)^{\ell}
$$

and

$$
\sum_{k=0}^{n} \frac{(-1)^{k}}{2^{k} k !}\left[\sum_{\ell=0}^{n-k} \frac{2^{\ell}}{\ell !}\left(\begin{array}{c}
n-k-1 \\
\ell-1
\end{array}\right) x^{2 \ell}\right] H_{k}^{2}(x)=\frac{1+(-1)^{n}}{2} \frac{(n-1) ! !}{n ! !} .
$$

\section{LEMMAS}

In order to prove our main results, we need several lemmas below.

Lemma 2.1 (2, p. 134, Theorem A] and [2, p. 139, Theorem C]). For $n \geq k \geq 0$, the Bell polynomials of the second kind, or say, partial Bell polynomials, denoted by $\mathrm{B}_{n, k}\left(x_{1}, x_{2}, \ldots, x_{n-k+1}\right)$, are defined by

$$
\mathrm{B}_{n, k}\left(x_{1}, x_{2}, \ldots, x_{n-k+1}\right)=\sum_{\substack{1 \leq i \leq n, \ell_{i} \in\{0\} \cup \mathbb{N} \\
\sum_{\begin{subarray}{c}{n=1 \\
i} }}^{n} i \ell_{i}=n} \\
{\sum_{i=1} \ell_{i}=k}\end{subarray}} \frac{n !}{\prod_{i=1}^{n-k+1} \ell_{i} !} \prod_{i=1}^{n-k+1}\left(\frac{x_{i}}{i !}\right)^{\ell_{i}}
$$

The Faà di Bruno formula can be described in terms of the Bell polynomials of the second kind $\mathrm{B}_{n, k}\left(x_{1}, x_{2}, \ldots, x_{n-k+1}\right)$ by

$$
\frac{\mathrm{d}^{n}}{\mathrm{~d} t^{n}} f \circ h(t)=\sum_{k=0}^{n} f^{(k)}(h(t)) \mathrm{B}_{n, k}\left(h^{\prime}(t), h^{\prime \prime}(t), \ldots, h^{(n-k+1)}(t)\right) .
$$

Lemma 2.2 ([2, p. 135]). For complex numbers $a$ and $b$, we have

$$
\mathrm{B}_{n, k}\left(a b x_{1}, a b^{2} x_{2}, \ldots, a b^{n-k+1} x_{n-k+1}\right)=a^{k} b^{n} \mathrm{~B}_{n, k}\left(x_{1}, x_{2}, \ldots, x_{n-k+1}\right) .
$$

Lemma 2.3 (44, Theorem 4.1], 9, Eq. (2.8)], and [10, Section 3]). For $0 \leq k \leq n$, the Bell polynomials of the second kind $\mathrm{B}_{n, k}$ satisfy

$$
\mathrm{B}_{n, k}(x, 1,0, \ldots, 0)=\frac{1}{2^{n-k}} \frac{n !}{k !}\left(\begin{array}{c}
k \\
n-k
\end{array}\right) x^{2 k-n} .
$$

Lemma 2.4 ([2, p. 135, Theorem B] and [6, Theorem 1.1]). For $n \geq k \geq 0$, we have

$$
\mathrm{B}_{n, k}(1 !, 2 !, \ldots,(n-k+1) !)=\left(\begin{array}{c}
n-1 \\
k-1
\end{array}\right) \frac{n !}{k !} .
$$


Lemma 2.5. For $n \geq k \geq 0$, the Bell polynomials of the second kind

$$
\mathrm{B}_{n, k}\left(1 !\left[1-(-1)^{2}\right], 2 !\left[1-(-1)^{3}\right], \ldots,(n-k+1) !\left[1-(-1)^{n-k+2}\right]\right)
$$

satisfy

$$
\begin{gathered}
\mathrm{B}_{2 j+1, k}\left(1 !\left[1-(-1)^{2}\right], 2 !\left[1-(-1)^{3}\right], \ldots,(2 j-k+2) !\left[1-(-1)^{2 j-k+3}\right]\right)=0, \quad 2 j+1 \geq k, \\
\mathrm{~B}_{2 j, k}\left(1 !\left[1-(-1)^{2}\right], 2 !\left[1-(-1)^{3}\right], \ldots,(2 j-k+1) !\left[1-(-1)^{2 j-k+2}\right]\right)=0, \quad 2 j \geq k>j \geq 0,
\end{gathered}
$$
and

$$
\mathrm{B}_{2 j, k}\left(1 !\left[1-(-1)^{2}\right], 2 !\left[1-(-1)^{3}\right], \ldots,(2 j-k+1) !\left[1-(-1)^{2 j-k+2}\right]\right)=\frac{2^{k}(2 j) !}{k !}\left(\begin{array}{l}
j-1 \\
k-1
\end{array}\right)
$$

for $j \geq k \geq 0$. Equivalently and unifiedly,

$$
\mathrm{B}_{n, k}\left(1 !\left[1-(-1)^{2}\right], 2 !\left[1-(-1)^{3}\right], \ldots,(n-k+1) !\left[1-(-1)^{n-k+2}\right]\right)=\left[1+(-1)^{n}\right] \frac{2^{k-1} n !}{k !}\left(\begin{array}{c}
\frac{n}{2}-1 \\
k-1
\end{array}\right)
$$

or

$$
\mathrm{B}_{n, k}\left(0,2 !, 0,4 !, 0,6 !, 0,8 !, 0, \ldots, \frac{1-(-1)^{n-k+2}}{2}(n-k+1) !\right)=\frac{1+(-1)^{n}}{2} \frac{n !}{k !}\left(\begin{array}{c}
\frac{n}{2}-1 \\
k-1
\end{array}\right),
$$

where

$$
\left(\begin{array}{l}
\alpha \\
k
\end{array}\right)=\frac{\langle\alpha\rangle_{k}}{k !}= \begin{cases}\frac{1}{k !} \prod_{\ell=0}^{k-1}(\alpha-\ell+1), & k \in \mathbb{N} \\
1, & k=0\end{cases}
$$

for arbitrary $a \in \mathbb{C}$ and $k \geq 0$ and $\langle\alpha\rangle_{k}$ is called the falling factorial.

Proof. In [2, p. 133], it was listed that

$$
\frac{1}{k !}\left(\sum_{m=1}^{\infty} x_{m} \frac{t^{m}}{m !}\right)^{k}=\sum_{n=k}^{\infty} \mathrm{B}_{n, k}\left(x_{1}, x_{2}, \ldots, x_{n-k+1}\right) \frac{t^{n}}{n !}
$$

for $k \geq 0$. From this, it follows that

$$
\begin{gathered}
\sum_{n=k}^{\infty} \mathrm{B}_{n, k}\left(1 !\left[1-(-1)^{2}\right], 2 !\left[1-(-1)^{3}\right], \ldots,(n-k+1) !\left[1-(-1)^{n-k+2}\right]\right) \frac{t^{n}}{n !} \\
=\frac{1}{k !}\left[\sum_{m=1}^{\infty} 2 \cdot(2 m) ! \frac{t^{2 m}}{(2 m) !}\right]^{k}=\frac{2^{k}}{k !}\left(\frac{t^{2}}{1-t^{2}}\right)^{k}=\frac{2^{k}}{k !}\left(\frac{1}{1-t^{2}}-1\right)^{k} \\
=\frac{2^{k}}{k !} \sum_{\ell=0}^{k}(-1)^{k-\ell}\left(\begin{array}{c}
k \\
\ell
\end{array}\right)\left(\frac{1}{1-t^{2}}\right)^{\ell}=\frac{2^{k}}{k !} \sum_{\ell=0}^{k}(-1)^{k-\ell}\left(\begin{array}{c}
k \\
\ell
\end{array}\right) \frac{1}{\left(1-t^{2}\right)^{\ell}} .
\end{gathered}
$$

Further differentiating $m \geq k$ times and making use of (16), 17), and 18) yield

$$
\begin{aligned}
\sum_{n=m}^{\infty} \mathrm{B}_{n, k}\left(1 !\left[1-(-1)^{2}\right]\right. & \left., 2 !\left[1-(-1)^{3}\right], \ldots,(n-k+1) !\left[1-(-1)^{n-k+2}\right]\right)\langle n\rangle_{m} \frac{t^{n-m}}{n !} \\
= & \frac{2^{k}}{k !} \sum_{\ell=0}^{k}(-1)^{k-\ell}\left(\begin{array}{l}
k \\
\ell
\end{array}\right)\left[\frac{1}{\left(1-t^{2}\right)^{\ell}}\right]^{(m)}
\end{aligned}
$$




$$
\begin{aligned}
& =\frac{2^{k}}{k !} \sum_{\ell=0}^{k}(-1)^{k-\ell}\left(\begin{array}{l}
k \\
\ell
\end{array}\right) \sum_{p=0}^{m}\left(\frac{1}{u^{\ell}}\right)^{(p)} \mathrm{B}_{m, p}(-2 t,-2,0, \ldots, 0) \\
= & \frac{2^{k}}{k !} \sum_{\ell=0}^{k}(-1)^{k-\ell}\left(\begin{array}{c}
k \\
\ell
\end{array}\right) \sum_{p=0}^{m} \frac{(-1)^{p}\langle-\ell\rangle_{p}}{u^{\ell+p}}(-2)^{p} \mathrm{~B}_{m, p}(t, 1,0, \ldots, 0) \\
= & \frac{2^{k}}{k !} \sum_{\ell=0}^{k}(-1)^{k-\ell}\left(\begin{array}{c}
k \\
\ell
\end{array}\right) \sum_{p=0}^{m} \frac{2^{p}\langle-\ell\rangle_{p}}{\left(1-t^{2}\right)^{\ell+p}} \frac{1}{2^{m-p}} \frac{m !}{p !}\left(\begin{array}{c}
p \\
m-p
\end{array}\right) t^{2 p-m} .
\end{aligned}
$$

Taking $t \rightarrow 0$ gives

$$
\begin{gathered}
\mathrm{B}_{m, k}\left(1 !\left[1-(-1)^{2}\right], 2 !\left[1-(-1)^{3}\right], \ldots,(m-k+1) !\left[1-(-1)^{m-k+2}\right]\right) \\
=\frac{2^{k}}{k !} \sum_{\ell=0}^{k}(-1)^{k-\ell}\left(\begin{array}{c}
k \\
\ell
\end{array}\right) \lim _{t \rightarrow 0} \sum_{p=0}^{m} \frac{2^{p}\langle-\ell\rangle_{p}}{2^{m-p}} \frac{m !}{p !}\left(\begin{array}{c}
p \\
m-p
\end{array}\right) t^{2 p-m} \\
= \begin{cases}0, & m=2 j+1 \\
\frac{2^{k}}{k !} \sum_{\ell=0}^{k}(-1)^{k-\ell}\left(\begin{array}{c}
k \\
\ell
\end{array}\right)\langle-\ell\rangle_{j} \frac{(2 j) !}{j !}, & m=2 j\end{cases} \\
= \begin{cases}0, & m=2 j+1 \\
(-1)^{k} \frac{2^{k}}{k !}(2 j) ! \sum_{\ell=0}^{k}(-1)^{\ell}\left(\begin{array}{c}
k \\
\ell
\end{array}\right)\left(\begin{array}{c}
\ell+j-1 \\
j
\end{array}\right), & m=2 j\end{cases}
\end{gathered}
$$

which is equivalent to 20 and

$$
\begin{gathered}
\mathrm{B}_{2 j, k}\left(1 !\left[1-(-1)^{2}\right], 2 !\left[1-(-1)^{3}\right], \ldots,(2 j-k+1) !\left[1-(-1)^{2 j-k+2}\right]\right) \\
=(-1)^{k} \frac{2^{k}}{k !}(2 j) ! \sum_{\ell=0}^{k}(-1)^{\ell}\left(\begin{array}{c}
k \\
\ell
\end{array}\right)\left(\begin{array}{c}
\ell+j-1 \\
j
\end{array}\right) \\
=(-1)^{k} \frac{2^{k}}{k !}(2 j) !(-1)^{k}\left(\begin{array}{c}
j-1 \\
k-1
\end{array}\right)=\frac{2^{k}(2 j) !}{k !}\left(\begin{array}{l}
j-1 \\
k-1
\end{array}\right)
\end{gathered}
$$

for $j, k \geq 0$. The formulas 21 and $(22)$ are thus proved.

It is straightforward to rewrite 20$], 21$, and 22 as either 23) or 24). The proof of Lemma 2.5 is complete.

Remark 2.1. By the formula

$$
\begin{aligned}
\mathrm{B}_{n, k}\left(x_{1}+y_{1}, x_{2}+y_{2}, \ldots,\right. & \left.x_{n-k+1}+y_{n-k+1}\right) \\
& =\sum_{r+s=k} \sum_{\ell+m=n}\left(\begin{array}{l}
n \\
\ell
\end{array}\right) \mathrm{B}_{\ell, r}\left(x_{1}, x_{2}, \ldots, x_{\ell-r+1}\right) \mathrm{B}_{m, s}\left(y_{1}, y_{2}, \ldots, y_{m-s+1}\right)
\end{aligned}
$$

in [1, Example 2.6], [2, p. 136, Eq. [3n]], and [8, Lemma 5] and by the formulas (17) and (19), it follows that

$$
\begin{gathered}
\mathrm{B}_{n, k}\left(1 !\left[1-(-1)^{2}\right], 2 !\left[1-(-1)^{3}\right], \ldots,(n-k+1) !\left[1-(-1)^{n-k+2}\right]\right) \\
=\sum_{r+s=k} \sum_{\ell+m=n}\left(\begin{array}{l}
n \\
\ell
\end{array}\right) \mathrm{B}_{\ell, r}(1 !, 2 !, \ldots,(\ell-r+1) !) \mathrm{B}_{m, s}\left(-1 !, 2 !, \ldots,(-1)^{m-s+1}(m-s+1) !\right)
\end{gathered}
$$




$$
\begin{gathered}
=\sum_{r+s=k} \sum_{\ell+m=n}\left(\begin{array}{l}
n \\
\ell
\end{array}\right)\left(\begin{array}{l}
\ell-1 \\
r-1
\end{array}\right) \frac{\ell !}{r !}(-1)^{m} \mathrm{~B}_{m, s}(1 !, 2 !, \ldots,(m-s+1) !) \\
=\sum_{r+s=k} \sum_{\ell+m=n}(-1)^{m}\left(\begin{array}{l}
n \\
\ell
\end{array}\right)\left(\begin{array}{l}
\ell-1 \\
r-1
\end{array}\right) \frac{\ell !}{r !}\left(\begin{array}{c}
m-1 \\
s-1
\end{array}\right) \frac{m !}{s !} \\
=\sum_{r=0}^{k} \sum_{\ell=0}^{n}(-1)^{n-\ell}\left(\begin{array}{l}
n \\
\ell
\end{array}\right)\left(\begin{array}{l}
\ell-1 \\
r-1
\end{array}\right)\left(\begin{array}{l}
n-\ell-1 \\
k-r-1
\end{array}\right) \frac{\ell !(n-\ell) !}{r !(k-r) !} \\
=\frac{n !}{k !} \sum_{r=0}^{k} \sum_{\ell=0}^{n}(-1)^{n-\ell}\left(\begin{array}{l}
k \\
r
\end{array}\right)\left(\begin{array}{l}
\ell-1 \\
r-1
\end{array}\right)\left(\begin{array}{l}
n-\ell-1 \\
k-r-1
\end{array}\right)
\end{gathered}
$$

which is not simpler than the nice expression $(23)$.

\section{Proofs of MAIN RESUlts}

Now we are in a position to prove our main results.

Proof of Theorem 1.1. By the formulas (16), 17), and (18), we obtain

$$
\begin{aligned}
\frac{\mathrm{d}^{k}}{\mathrm{~d} t^{k}}\left(\frac{1}{\sqrt{1-t^{2}}}\right) & =\sum_{\ell=0}^{k} \frac{\mathrm{d}^{\ell}}{\mathrm{d} u^{\ell}}\left(\frac{1}{\sqrt{u}}\right) \mathrm{B}_{k, \ell}(-2 t,-2,0, \ldots, 0) \\
& =\sum_{\ell=0}^{k}\left\langle-\frac{1}{2}\right\rangle_{\ell} \frac{1}{u^{\ell+1 / 2}}(-2)^{\ell} \mathrm{B}_{k, \ell}(t, 1,0, \ldots, 0) \\
& =\sum_{\ell=0}^{k}\left\langle-\frac{1}{2}\right\rangle_{\ell} \frac{1}{\left(1-t^{2}\right)^{\ell+1 / 2}}(-2)^{\ell} \frac{1}{2^{k-\ell}} \frac{k !}{\ell !}\left(\begin{array}{c}
\ell \\
k-\ell
\end{array}\right) t^{2 \ell-k} \\
& =\sum_{\ell=0}^{k} \frac{(2 \ell-1) ! !}{2^{\ell}} \frac{1}{\left(1-t^{2}\right)^{\ell+1 / 2}} 2^{\ell} \frac{1}{2^{k-\ell}} \frac{k !}{\ell !}\left(\begin{array}{c}
\ell \\
k-\ell
\end{array}\right) t^{2 \ell-k} \\
& =\frac{1}{\sqrt{1-t^{2}}} \frac{k !}{(2 t)^{k}} \sum_{\ell=0}^{k} \frac{(2 \ell-1) ! ! 2^{\ell}}{\ell !}\left(\begin{array}{c}
\ell \\
k-\ell
\end{array}\right) \frac{t^{2 \ell}}{\left(1-t^{2}\right)^{\ell}},
\end{aligned}
$$

where $u=u(t)=1-t^{2}$.

Similarly, by the formulas $(16,, 17)$, and 190 , we acquire

$$
\begin{aligned}
\frac{\mathrm{d}^{k}}{\mathrm{~d} t^{k}}\left(\exp \frac{x t}{1+t}\right) & =\sum_{\ell=0}^{k} x^{\ell} e^{x v} \mathrm{~B}_{k, \ell}\left(\frac{1 !}{(1+t)^{2}}, \frac{-2 !}{(1+t)^{3}}, \ldots, \frac{(-1)^{k-\ell}(k-\ell+1) !}{(1+t)^{k-\ell+2}}\right) \\
& =\sum_{\ell=0}^{k} x^{\ell} e^{x t /(1+t)} \frac{(-1)^{k+\ell}}{(1+t)^{k+\ell}} \mathrm{B}_{k, \ell}(1 !, 2 !, \ldots,(k-\ell+1) !) \\
& =e^{x t /(1+t)} \frac{(-1)^{k} k !}{(1+t)^{k}} \sum_{\ell=0}^{k} \frac{(-1)^{\ell}}{\ell !}\left(\begin{array}{c}
k-1 \\
\ell-1
\end{array}\right) \frac{x^{\ell}}{(1+t)^{\ell}},
\end{aligned}
$$


where $v=v(t)=\frac{t}{1+t}$. Making use of the above two results and employing the Leibniz rule yield

$$
\begin{aligned}
\frac{\mathrm{d}^{n} F(t)}{\mathrm{d} t^{n}}= & \sum_{k=0}^{n}\left(\begin{array}{l}
n \\
k
\end{array}\right) \frac{\mathrm{d}^{k}}{\mathrm{~d} t^{k}}\left(\frac{1}{\sqrt{1-t^{2}}}\right) \frac{\mathrm{d}^{n-k}}{\mathrm{~d} t^{n-k}}\left(\exp \frac{x t}{1+t}\right) \\
= & \sum_{k=0}^{n}\left(\begin{array}{l}
n \\
k
\end{array}\right) \frac{1}{\sqrt{1-t^{2}}} \frac{k !}{(2 t)^{k}} \sum_{\ell=0}^{k} \frac{(2 \ell-1) ! ! 2^{\ell}}{\ell !}\left(\begin{array}{c}
\ell \\
k-\ell
\end{array}\right) \frac{t^{2 \ell}}{\left(1-t^{2}\right)^{\ell}} \\
& \times e^{x t /(1+t)} \frac{(-1)^{n-k}(n-k) !}{(1+t)^{n-k}} \sum_{\ell=0}^{n-k} \frac{(-1)^{\ell}}{\ell !}\left(\begin{array}{c}
n-k-1 \\
\ell-1
\end{array}\right) \frac{x^{\ell}}{(1+t)^{\ell}} \\
= & \frac{e^{x t /(1+t)}}{\sqrt{1-t^{2}}} \frac{(-1)^{n} n !}{(1+t)^{n}} \sum_{k=0}^{n} \frac{(-1)^{k}(1+t)^{k}}{(2 t)^{k}} \sum_{\ell=0}^{k} \frac{(2 \ell-1) ! ! 2^{\ell}}{\ell !} \\
& \times\left(\begin{array}{c}
\ell \\
k-\ell
\end{array}\right) \frac{t^{2 \ell}}{\left(1-t^{2}\right)^{\ell}} \sum_{m=0}^{n-k} \frac{(-1)^{m}}{m !}\left(\begin{array}{c}
n-k-1 \\
m-1
\end{array}\right) \frac{x^{m}}{(1+t)^{m}} \\
= & F(t) \frac{(-1)^{n} n !}{(1+t)^{n}} \sum_{k=0}^{n} \frac{(-1)^{k}(1+t)^{k}}{2^{k} t^{k}} \sum_{\ell=0}^{k} \frac{(2 \ell-1) ! ! 2^{\ell}}{\ell !}\left(\begin{array}{c}
\ell \\
k-\ell
\end{array}\right) \\
& \times \frac{t^{2 \ell}}{\left(1-t^{2}\right)^{\ell}} \sum_{m=0}^{n-k} \frac{(-1)^{m}}{m !}\left(\begin{array}{c}
n-k-1 \\
m-1
\end{array}\right) \frac{x^{m}}{(1+t)^{m}} \\
= & F(t) \frac{(-1)^{n} n !}{(1+t)^{n}} \sum_{m=0}^{n} \frac{(-1)^{m}}{m !} \frac{1}{(1+t)^{m}}\left(\begin{array}{c}
n-m \\
k=0
\end{array} \frac{(-1)^{k}(1+t)^{k}}{2^{k}}\right. \\
& \left.\times\left(\begin{array}{c}
n-k-1 \\
m-1
\end{array}\right)\left[\frac{1}{t^{k}} \sum_{\ell=0}^{k} \frac{(2 \ell-1) ! ! 2^{\ell}}{\ell !}\left(\begin{array}{c}
\ell \\
k-\ell
\end{array}\right) \frac{t^{2 \ell}}{\left(1-t^{2}\right)^{\ell}}\right]\right) x^{m} .
\end{aligned}
$$

The formula (6) is thus proved. The proof of Theorem 1.1 is complete.

Proof of Theorem 1.2. Since

$$
\begin{gathered}
{\left[\frac{t^{2 \ell}}{\left(1-t^{2}\right)^{\ell}}\right]^{(k)}=\left[\left(\frac{1}{2(t+1)}-\frac{1}{2(t-1)}-1\right)^{\ell}\right]^{(k)}} \\
=\sum_{p=0}^{k}\left(w^{\ell}\right)^{(p)} \mathrm{B}_{k, p}\left(-\frac{1 !}{2}\left[\frac{1}{(t+1)^{2}}-\frac{1}{(t-1)^{2}}\right], \frac{2 !}{2}\left[\frac{1}{(t+1)^{3}}-\frac{1}{(t-1)^{3}}\right],\right. \\
\left.\ldots,(-1)^{k-p+1} \frac{(k-p+1) !}{2}\left[\frac{1}{(t+1)^{k-p+2}}-\frac{1}{(t-1)^{k-p+2}}\right]\right) \\
=\sum_{p=0}^{k}\langle\ell\rangle_{p} w^{\ell-p} \frac{(-1)^{k}}{2^{p}} \mathrm{~B}_{k, p}\left(1 !\left[\frac{1}{(t+1)^{2}}-\frac{1}{(t-1)^{2}}\right], 2 !\left[\frac{1}{(t+1)^{3}}-\frac{1}{(t-1)^{3}}\right],\right. \\
\left.\ldots,(k-p+1) !\left[\frac{1}{(t+1)^{k-p+2}}-\frac{1}{(t-1)^{k-p+2}}\right]\right)
\end{gathered}
$$




$$
\begin{gathered}
=\sum_{p=0}^{k}\langle\ell\rangle_{p}\left(\frac{t^{2}}{1-t^{2}}\right)^{\ell-p} \frac{(-1)^{k}}{2^{p}} \mathrm{~B}_{k, p}\left(1 !\left[\frac{1}{(t+1)^{2}}-\frac{1}{(t-1)^{2}}\right],\right. \\
\left.2 !\left[\frac{1}{(t+1)^{3}}-\frac{1}{(t-1)^{3}}\right], \ldots,(k-p+1) !\left[\frac{1}{(t+1)^{k-p+2}}-\frac{1}{(t-1)^{k-p+2}}\right]\right) \\
\rightarrow\langle\ell\rangle_{\ell} \frac{(-1)^{k}}{2^{\ell}} \mathrm{B}_{k, \ell}\left(1 !\left[1-(-1)^{2}\right], 2 !\left[1-(-1)^{3}\right], \ldots,(k-\ell+1) !\left[1-(-1)^{k-\ell+2}\right]\right) \\
=(-1)^{k} \frac{\ell !}{2^{\ell}} \mathrm{B}_{k, \ell}\left(1 !\left[1-(-1)^{2}\right], 2 !\left[1-(-1)^{3}\right], \ldots,(k-\ell+1) !\left[1-(-1)^{k-\ell+2}\right]\right)
\end{gathered}
$$

as $t \rightarrow 0$, where $w=w(t)=\frac{t^{2}}{1-t^{2}}=\frac{1}{2(t+1)}-\frac{1}{2(t-1)}-1$, employing the L'Hôspital rule and the formula 23) leads to

$$
\begin{gathered}
\lim _{t \rightarrow 0}\left[\frac{1}{t^{k}} \sum_{\ell=0}^{k} \frac{(2 \ell-1) ! ! 2^{\ell}}{\ell !}\left(\begin{array}{c}
\ell \\
k-\ell
\end{array}\right) \frac{t^{2 \ell}}{\left(1-t^{2}\right)^{\ell}}\right]=\frac{1}{k !} \sum_{\ell=0}^{k} \frac{(2 \ell-1) ! ! 2^{\ell}}{\ell !}\left(\begin{array}{c}
\ell \\
k-\ell
\end{array}\right) \lim _{t \rightarrow 0}\left[\frac{t^{2 \ell}}{\left(1-t^{2}\right)^{\ell}}\right]^{(k)} \\
=\frac{(-1)^{k}}{k !} \sum_{\ell=0}^{k}(2 \ell-1) ! !\left(\begin{array}{c}
\ell \\
k-\ell
\end{array}\right) \mathrm{B}_{k, \ell}\left(1 !\left[1-(-1)^{2}\right], 2 !\left[1-(-1)^{3}\right], \ldots,(k-\ell+1) !\left[1-(-1)^{k-\ell+2}\right]\right) \\
=\frac{(-1)^{k}}{k !} \sum_{\ell=0}^{k}(2 \ell-1) ! !\left(\begin{array}{c}
\ell \\
k-\ell
\end{array}\right)\left[1+(-1)^{k}\right] \frac{2^{\ell-1} k !}{\ell !}\left(\begin{array}{c}
\frac{k}{2}-1 \\
\ell-1
\end{array}\right) \\
=(-1)^{k}\left[1+(-1)^{k}\right] \sum_{\ell=0}^{k} \frac{2^{\ell-1}(2 \ell-1) ! !}{\ell !}\left(\begin{array}{c}
\ell \\
k-\ell
\end{array}\right)\left(\begin{array}{c}
\frac{k}{2}-1 \\
\ell-1
\end{array}\right) \\
=\frac{1+(-1)^{k}}{2} \sum_{\ell=0}^{k} \frac{2^{2 \ell}(2 \ell-1) ! !}{(2 \ell) ! !}\left(\begin{array}{c}
\ell \\
k-\ell
\end{array}\right)\left(\begin{array}{c}
\frac{k}{2}-1 \\
\ell-1
\end{array}\right)=\frac{1+(-1)^{k}}{2} \frac{2^{k}(k-1) ! !}{k ! !} .
\end{gathered}
$$

Therefore, taking the limit $t \rightarrow$ on both sides of (6) yields

$$
\begin{aligned}
\lim _{t \rightarrow 0} \frac{\mathrm{d}^{n} F(t)}{\mathrm{d} t^{n}}= & (-1)^{n} n ! \sum_{m=0}^{n} \frac{(-1)^{m}}{m !} \sum_{k=0}^{n-m} \frac{(-1)^{k}}{2^{k}}\left(\begin{array}{c}
n-k-1 \\
m-1
\end{array}\right) \\
& \times \lim _{t \rightarrow 0}\left[\frac{1}{t^{k}} \sum_{\ell=0}^{k} \frac{(2 \ell-1) ! ! 2^{\ell}}{\ell !}\left(\begin{array}{c}
\ell \\
k-\ell
\end{array}\right) \frac{t^{2 \ell}}{\left(1-t^{2}\right)^{\ell}}\right] x^{m} \\
= & (-1)^{n} n ! \sum_{m=0}^{n} \frac{(-1)^{m}}{m !} \sum_{k=0}^{n-m} \frac{(-1)^{k}}{2^{k}}\left(\begin{array}{c}
n-k-1 \\
m-1
\end{array}\right) \frac{1+(-1)^{k}}{2} \frac{2^{k}(k-1) ! !}{k ! !} x^{m} \\
= & (-1)^{n} n ! \sum_{m=0}^{n} \frac{(-1)^{m}}{m !}\left[\sum_{k=0}^{n-m}\left(\begin{array}{c}
n-k-1 \\
m-1
\end{array}\right) \frac{1+(-1)^{k}}{2} \frac{(k-1) ! !}{k ! !}\right] x^{m}
\end{aligned}
$$

which means by $(3)$ that

$$
\frac{H_{n}^{2}(\sqrt{x / 2})}{2^{n}}=(-1)^{n} n ! \sum_{m=0}^{n} \frac{(-1)^{m}}{m !}\left[\sum_{k=0}^{n-m} \frac{1+(-1)^{k}}{2} \frac{(k-1) ! !}{k ! !}\left(\begin{array}{c}
n-k-1 \\
m-1
\end{array}\right)\right] x^{m}, \quad n \geq 0 .
$$

This can be rearranged as (7). The proof of Theorem 1.2 is complete. 
Proof of Theorem 1.3. By the formulas (16), 17), and (18), we obtain

$$
\begin{aligned}
\frac{\mathrm{d}^{n} e^{2 x t-t^{2}}}{\mathrm{~d} t^{n}} & =\sum_{k=0}^{n}\left(e^{u}\right)^{(k)} \mathrm{B}_{n, k}(2 x-2 t,-2,0, \ldots, 0) \\
& =\sum_{k=0}^{n} e^{2 x t-t^{2}}(-2)^{k} \mathrm{~B}_{n, k}(t-x, 1,0, \ldots, 0) \\
& =e^{2 x t-t^{2}} \sum_{k=0}^{n}(-2)^{k} \frac{1}{2^{n-k}} \frac{n !}{k !}\left(\begin{array}{c}
k \\
n-k
\end{array}\right)(t-x)^{2 k-n} \\
& =\frac{n !}{2^{n}} \frac{e^{2 x t-t^{2}}}{(t-x)^{n}} \sum_{k=0}^{n}(-1)^{k} \frac{2^{2 k}}{k !}\left(\begin{array}{c}
k \\
n-k
\end{array}\right)(t-x)^{2 k}
\end{aligned}
$$

where $u=u(t)=2 x t-t^{2}$. Hence, we acquire

$$
\begin{aligned}
H_{n}(x) & =\lim _{t \rightarrow 0} \frac{\mathrm{d}^{n} e^{2 x t-t^{2}}}{\mathrm{~d} t^{n}} \\
& =\frac{n !}{2^{n}} \lim _{t \rightarrow 0} \frac{e^{2 x t-t^{2}}}{(t-x)^{n}} \sum_{k=0}^{n}(-1)^{k} \frac{2^{2 k}}{k !}\left(\begin{array}{c}
k \\
n-k
\end{array}\right)(t-x)^{2 k} \\
& =\frac{n !}{2^{n}} \frac{1}{(-x)^{n}} \sum_{k=0}^{n}(-1)^{k} \frac{2^{2 k}}{k !}\left(\begin{array}{c}
k \\
n-k
\end{array}\right)(-x)^{2 k} \\
& =(-1)^{n} \frac{n !}{2^{n}} \sum_{k=0}^{n}(-1)^{k} \frac{2^{2 k}}{k !}\left(\begin{array}{c}
k \\
n-k
\end{array}\right) x^{2 k-n} .
\end{aligned}
$$

The formula $(8)$ follows. This formula can also be derived similarly by considering

$$
H_{n}(x)=(-1)^{n} e^{x^{2}} \frac{\mathrm{d}^{n}}{\mathrm{~d} x^{n}} e^{-x^{2}} \quad \text { or } \quad H_{n}(x)=e^{x^{2} / 2}\left(x-\frac{\mathrm{d}}{\mathrm{d} x}\right)^{n} e^{-x^{2} / 2} .
$$

The proof of Theorem 1.3 is complete.

Proof of Theorem 1.4. Differentiating with respect to $x$ on both sides of (1) yields

$$
\begin{gathered}
2 t e^{2 x t-t^{2}}=\sum_{n=0}^{\infty} H_{n}^{\prime}(x) \frac{t^{n}}{n !}, \\
2 t \sum_{n=0}^{\infty} H_{n}(x) \frac{t^{n}}{n !}=\sum_{n=0}^{\infty} H_{n}^{\prime}(x) \frac{t^{n}}{n !}, \\
\sum_{n=0}^{\infty} 2 H_{n}(x) \frac{t^{n+1}}{n !}=\sum_{n=0}^{\infty} H_{n}^{\prime}(x) \frac{t^{n}}{n !}, \\
\sum_{n=1}^{\infty} 2 H_{n-1}(x) \frac{t^{n}}{(n-1) !}=\sum_{n=0}^{\infty} H_{n}^{\prime}(x) \frac{t^{n}}{n !} .
\end{gathered}
$$

Hence, it follows that $H_{0}^{\prime}(x)=0$ and the formula $(9)$ is valid. 
Differentiating with respect to $x$ on both sides of (2) gives

$$
\begin{gathered}
\frac{4 x t}{1+t} \frac{1}{\sqrt{1-t^{2}}} \exp \frac{2 x^{2} t}{1+t}=\sum_{n=0}^{\infty} \frac{2 H_{n}(x) H_{n}^{\prime}(x)}{2^{n}} \frac{t^{n}}{n !}, \\
\frac{2 x t}{1+t} \sum_{n=0}^{\infty} \frac{H_{n}^{2}(x)}{2^{n}} \frac{t^{n}}{n !}=\sum_{n=0}^{\infty} \frac{H_{n}(x) H_{n}^{\prime}(x)}{2^{n}} \frac{t^{n}}{n !}, \\
2 x t \sum_{n=0}^{\infty} \frac{H_{n}^{2}(x)}{2^{n}} \frac{t^{n}}{n !}=(1+t) \sum_{n=0}^{\infty} \frac{H_{n}(x) H_{n}^{\prime}(x)}{2^{n}} \frac{t^{n}}{n !}, \\
\sum_{n=0}^{\infty} \frac{2 x H_{n}^{2}(x)}{2^{n}} \frac{t^{n+1}}{n !}=\sum_{n=0}^{\infty} \frac{H_{n}(x) H_{n}^{\prime}(x)}{2^{n}} \frac{t^{n}}{n !}+\sum_{n=0}^{\infty} \frac{H_{n}(x) H_{n}^{\prime}(x)}{2^{n}} \frac{t^{n+1}}{n !}, \\
\sum_{n=1}^{\infty} \frac{2 x H_{n-1}^{2}(x)}{2^{n-1}} \frac{t^{n}}{(n-1) !}=\sum_{n=0}^{\infty} \frac{H_{n}(x) H_{n}^{\prime}(x)}{2^{n}} \frac{t^{n}}{n !}+\sum_{n=1}^{\infty} \frac{H_{n-1}(x) H_{n-1}^{\prime}(x)}{2^{n-1}} \frac{t^{n}}{(n-1) !} .
\end{gathered}
$$

This means that $H_{0}^{\prime}(x)=0$ and

$$
\frac{2 x H_{n-1}^{2}(x)}{2^{n-1}} \frac{t^{n}}{(n-1) !}=\frac{H_{n}(x) H_{n}^{\prime}(x)}{2^{n}} \frac{t^{n}}{n !}+\frac{H_{n-1}(x) H_{n-1}^{\prime}(x)}{2^{n-1}} \frac{t^{n}}{(n-1) !}
$$

for $n \in \mathbb{N}$, which can be simplified as

$$
H_{n}(x) H_{n}^{\prime}(x)=2 n H_{n-1}(x)\left[2 x H_{n-1}(x)-H_{n-1}^{\prime}(x)\right]
$$

Combining this with (9) derives the formula 100 .

Substituting (9) into (10) results in (11) readily. The proof of Theorem 1.4 is complete.

Proof of Theorem 1.5. It is easy to see that $e^{t^{2}} e^{2 x t-t^{2}}=e^{2 x t}$. Differentiating with respect to $t$ on both sides of this equation and utilizing (16), (17), and (18) give

$$
\begin{gathered}
\sum_{k=0}^{n}\left(\begin{array}{l}
n \\
k
\end{array}\right) \frac{\mathrm{d}^{n-k} e^{t^{2}}}{\mathrm{~d} t^{n-k}} \frac{\mathrm{d}^{k} e^{2 x t-t^{2}}}{\mathrm{~d} t^{k}}=(2 x)^{n} e^{2 x t}, \\
\sum_{k=0}^{n}\left(\begin{array}{l}
n \\
k
\end{array}\right) \sum_{\ell=0}^{n-k} e^{t^{2}} \mathrm{~B}_{n-k, \ell}(2 t, 2,0, \ldots, 0) \frac{\mathrm{d}^{k} e^{2 x t-t^{2}}}{\mathrm{~d} t^{k}}=(2 x)^{n} e^{2 x t}, \\
e^{t^{2}} \sum_{k=0}^{n}\left(\begin{array}{l}
n \\
k
\end{array}\right) \sum_{\ell=0}^{n-k} 2^{\ell} \frac{1}{2^{n-k-\ell}} \frac{(n-k) !}{\ell !}\left(\begin{array}{c}
\ell \\
n-k-\ell
\end{array}\right) t^{2 \ell-n+k} \frac{\mathrm{d}^{k} e^{2 x t-t^{2}}}{\mathrm{~d} t^{k}}=(2 x)^{n} e^{2 x t}, \\
e^{t^{2}} \sum_{k=0}^{n}\left(\begin{array}{l}
n \\
k
\end{array}\right) \frac{(n-k) !}{2^{n-k}} \sum_{\ell=0}^{n-k} \frac{2^{2 \ell}}{\ell !}\left(\begin{array}{c}
\ell \\
n-k-\ell
\end{array}\right) t^{2 \ell-n+k} \frac{\mathrm{d}^{k} e^{2 x t-t^{2}}}{\mathrm{~d} t^{k}}=(2 x)^{n} e^{2 x t} .
\end{gathered}
$$

Further taking the limit $t \rightarrow 0$ yields

$$
\begin{gathered}
\sum_{k=0}^{n}\left(\begin{array}{l}
n \\
k
\end{array}\right) \frac{(n-k) !}{2^{n-k}} \frac{1+(-1)^{n-k}}{2} \frac{2^{3(n-k) / 2}}{(n-k) ! !} \lim _{t \rightarrow 0} \frac{\mathrm{d}^{k} e^{2 x t-t^{2}}}{\mathrm{~d} t^{k}}=(2 x)^{n}, \\
n ! \sum_{k=0}^{n} \frac{1+(-1)^{n-k}}{2} \frac{2^{(n-k) / 2}}{(n-k) ! ! k !} H_{k}(x)=(2 x)^{n}
\end{gathered}
$$

The recurrence relation 12 is thus proved. 
Similarly, from $e^{-2 x t} e^{2 x t-t^{2}}=e^{-t^{2}}$, it follows that

$$
\begin{gathered}
\sum_{k=0}^{n}\left(\begin{array}{l}
n \\
k
\end{array}\right)(-2 x)^{n-k} e^{-2 x t} \frac{\mathrm{d}^{k} e^{2 x t-t^{2}}}{\mathrm{~d} t^{k}}=\sum_{k=0}^{n} e^{-t^{2}} \mathrm{~B}_{n, k}(-2 t,-2,0, \ldots, 0), \\
\sum_{k=0}^{n}\left(\begin{array}{l}
n \\
k
\end{array}\right)(-2 x)^{n-k} e^{-2 x t} \frac{\mathrm{d}^{k} e^{2 x t-t^{2}}}{\mathrm{~d} t^{k}}=\sum_{k=0}^{n} e^{-t^{2}}(-2)^{k} \frac{1}{2^{n-k}} \frac{n !}{k !}\left(\begin{array}{c}
k \\
n-k
\end{array}\right) t^{2 k-n},
\end{gathered}
$$

and, as $t \rightarrow 0$,

$$
\sum_{k=0}^{n}\left(\begin{array}{l}
n \\
k
\end{array}\right)(-2 x)^{n-k} H_{k}(x)=\lim _{t \rightarrow 0} \frac{1}{2^{n}} \sum_{k=0}^{n}(-1)^{k} \frac{2^{3 k} n !}{(2 k) ! !}\left(\begin{array}{c}
k \\
n-k
\end{array}\right) t^{2 k-n} .
$$

The recurrence relation 13 is thus proved.

Similarly, since

and

$$
\sqrt{1-t^{2}} \frac{1}{\sqrt{1-t^{2}}} \exp \frac{2 x^{2} t}{1+t}=\exp \frac{2 x^{2} t}{1+t}
$$

$$
\exp \frac{-2 x^{2} t}{1+t} \frac{1}{\sqrt{1-t^{2}}} \exp \frac{2 x^{2} t}{1+t}=\frac{1}{\sqrt{1-t^{2}}},
$$

by $25,, 26),(18)$, the formula

$$
\begin{aligned}
\left(\sqrt{1-t^{2}}\right)^{(k)} & =\sum_{\ell=0}^{k}\left\langle\frac{1}{2}\right\rangle_{\ell} u^{1 / 2-\ell} \mathrm{B}_{k, \ell}(-2 t,-2,0, \ldots, 0) \\
& =\sum_{\ell=0}^{k} \frac{(-1)^{\ell-1}(2 \ell-3) ! !}{2^{\ell}}\left(1-t^{2}\right)^{1 / 2-\ell}(-2)^{\ell} \mathrm{B}_{k, \ell}(t, 1,0, \ldots, 0) \\
& =-\sum_{\ell=0}^{k}(2 \ell-3) ! !\left(1-t^{2}\right)^{1 / 2-\ell} \frac{1}{2^{k-\ell}} \frac{k !}{\ell !}\left(\begin{array}{c}
\ell \\
k-\ell
\end{array}\right) t^{2 \ell-k} \\
& =-\frac{k !}{2^{k}} \frac{\left(1-t^{2}\right)^{1 / 2}}{t^{k}} \sum_{\ell=0}^{k}(2 \ell-3) ! ! \frac{2^{\ell}}{\ell !}\left(\begin{array}{c}
\ell \\
k-\ell
\end{array}\right) \frac{t^{2 \ell}}{\left(1-t^{2}\right)^{\ell}},
\end{aligned}
$$

and by the Leibniz rule for differentiation, it follows that

$$
\begin{gathered}
\sum_{k=0}^{n}\left(\begin{array}{l}
n \\
k
\end{array}\right)\left(\sqrt{1-t^{2}}\right)^{(n-k)}\left(\frac{1}{\sqrt{1-t^{2}}} \exp \frac{2 x^{2} t}{1+t}\right)^{(k)}=\left(\exp \frac{2 x^{2} t}{1+t}\right)^{(n)} \\
-\sum_{k=0}^{n}\left(\begin{array}{l}
n \\
k
\end{array}\right) \frac{(n-k) !}{2^{n-k}} \frac{\left(1-t^{2}\right)^{1 / 2}}{t^{n-k}} \sum_{\ell=0}^{n-k}(2 \ell-3) ! ! \frac{2^{\ell}}{\ell !}\left(\begin{array}{c}
\ell \\
n-k-\ell
\end{array}\right) \frac{t^{2 \ell}}{\left(1-t^{2}\right)^{\ell}}\left(\frac{1}{\sqrt{1-t^{2}}} \exp \frac{2 x^{2} t}{1+t}\right)^{(k)} \\
=e^{2 x^{2} t /(1+t)} \frac{(-1)^{n} n !}{(1+t)^{n}} \sum_{\ell=0}^{n} \frac{(-1)^{\ell}}{\ell !}\left(\begin{array}{c}
n-1 \\
\ell-1
\end{array}\right) \frac{\left(2 x^{2}\right)^{\ell}}{(1+t)^{\ell}}
\end{gathered}
$$

and

$$
\sum_{k=0}^{n}\left(\begin{array}{l}
n \\
k
\end{array}\right)\left(\exp \frac{-2 x^{2} t}{1+t}\right)^{(n-k)}\left(\frac{1}{\sqrt{1-t^{2}}} \exp \frac{2 x^{2} t}{1+t}\right)^{(k)}=\left(\frac{1}{\sqrt{1-t^{2}}}\right)^{(n)}
$$




$$
\begin{gathered}
\sum_{k=0}^{n}\left(\begin{array}{l}
n \\
k
\end{array}\right) e^{-2 x^{2} t /(1+t)} \frac{(-1)^{n-k}(n-k) !}{(1+t)^{n-k}} \sum_{\ell=0}^{n-k} \frac{(-1)^{\ell}}{\ell !}\left(\begin{array}{c}
n-k-1 \\
\ell-1
\end{array}\right) \frac{\left(-2 x^{2}\right)^{\ell}}{(1+t)^{\ell}}\left(\frac{1}{\sqrt{1-t^{2}}} \exp \frac{2 x^{2} t}{1+t}\right)^{(k)} \\
=\frac{1}{\sqrt{1-t^{2}}} \frac{n !}{(2 t)^{n}} \sum_{\ell=0}^{n} \frac{(2 \ell-1) ! ! 2^{\ell}}{\ell !}\left(\begin{array}{c}
\ell \\
n-\ell
\end{array}\right) \frac{t^{2 \ell}}{\left(1-t^{2}\right)^{\ell}}
\end{gathered}
$$

Further taking the limit $t \rightarrow 0$ results in

$$
\begin{array}{r}
-\sum_{k=0}^{n}\left(\begin{array}{l}
n \\
k
\end{array}\right) \frac{(n-k) !}{2^{n-k}} \lim _{t \rightarrow 0}\left[\frac{1}{t^{n-k}} \sum_{\ell=0}^{n-k}(2 \ell-3) ! ! \frac{2^{\ell}}{\ell !}\left(\begin{array}{c}
\ell \\
n-k-\ell
\end{array}\right) \frac{t^{2 \ell}}{\left(1-t^{2}\right)^{\ell}}\right] \frac{H_{k}^{2}(x)}{2^{k}} \\
=(-1)^{n} n ! \sum_{\ell=0}^{n} \frac{(-1)^{\ell}}{\ell !}\left(\begin{array}{c}
n-1 \\
\ell-1
\end{array}\right)\left(2 x^{2}\right)^{\ell}
\end{array}
$$

and

$$
\begin{aligned}
& \sum_{k=0}^{n}\left(\begin{array}{l}
n \\
k
\end{array}\right)(-1)^{n-k}(n-k) ! \sum_{\ell=0}^{n-k} \frac{(-1)^{\ell}}{\ell !}\left(\begin{array}{c}
n-k-1 \\
\ell-1
\end{array}\right)\left(-2 x^{2}\right)^{\ell} \frac{H_{k}^{2}(x)}{2^{k}} \\
& \quad=n ! \lim _{t \rightarrow 0}\left[\frac{1}{(2 t)^{n}} \sum_{\ell=0}^{n} \frac{(2 \ell-1) ! ! 2^{\ell}}{\ell !}\left(\begin{array}{c}
\ell \\
n-\ell
\end{array}\right) \frac{t^{2 \ell}}{\left(1-t^{2}\right)^{\ell}}\right] .
\end{aligned}
$$

Substituting (27) into (28) and (29) acquires the recurrence relations (14) and (15). The proof of Theorem 1.5 is complete.

\section{REFERENCES}

[1] A. Aboud, J.-P. Bultel, A. Chouria, J.-G. Luque, O. Mallet, Bell polynomials in combinatorial Hopf algebras, arXiv preprint (2014), available online at http://arxiv.org/abs/1402.2960

[2] L. Comtet, Advanced Combinatorics: The Art of Finite and Infinite Expansions, Revised and Enlarged Edition, D. Reidel Publishing Co., Dordrecht and Boston, 1974.

[3] A. Erdélyi, W. Magnus, F. Oberhettinger, and F. G. Tricomi, Higher Transcendental Functions, Vol. III. Based on notes left by Harry Bateman. Reprint of the 1955 original. Robert E. Krieger Publishing Co., Inc., Melbourne, Fla., 1981.

[4] B.-N. Guo and F. Qi, Explicit formulas for special values of the Bell polynomials of the second kind and the Euler numbers, ResearchGate Technical Report (2015), available online at http://dx.doi.org/10.13140/2.1. 3794.8808

[5] T. Kim, D. S. Kim, L.-C. Jang, and H. I. Kwon, On differential equations associated with squared Hermite polynomials, J. Comput. Anal. Appl. 23 (2017), no. 5, 1252-1264.

[6] F. Qi, Diagonal recurrence relations for the Stirling numbers of the first kind, Contrib. Discrete Math. 11 (2016), 22-30; Available online at http://hdl.handle.net/10515/sy5wh2dx6 and http://dx.doi.org/10515/ sy5wh2dx6

[7] F. Qi and B.-N. Guo, Viewing some ordinary differential equations from the angle of derivative polynomials, Preprints 2016, 2016100043, 12 pages; Available online at http://dx.doi.org/10.20944/preprints201610. 0043.v1

[8] F. Qi, X.-T. Shi, and B.-N. Guo, Two explicit formulas of the Schröder numbers, Integers 16 (2016), Paper No. A23, 15 pages.

[9] F. Qi, X.-T. Shi, F.-F. Liu, and D. V. Kruchinin, Several formulas for special values of the Bell polynomials of the second kind and applications, J. Appl. Anal. Comput. (2017), in press. ResearchGate Technical Report (2015), available online at http://dx.doi.org/10.13140/RG.2.1.3230.1927

[10] F. Qi and M.-M. Zheng, Explicit expressions for a family of the Bell polynomials and applications, Appl. Math. Comput. 258 (2015), 597-607; Available online at http://dx.doi.org/10.1016/j.amc.2015.02.027 

access article distributed under the terms and conditions of the Creative Commons Attribution (CC BY) license http://creativecommons.org/licenses/by/4.0/).

$U R L:$ https://qifeng618. wordpress.com

$U R L:$ http://www.researchgate.net/profile/Bai-Ni_Guo/ 